\title{
Protection of Hesperidin against Methotrexate- Induced Nephrotoxicity may be Mediated by Nrf2/H0-1 Pathway
}

\author{
Mohamed Aly Morsy ${ }^{1,2, *}$, Azza Ali Kamel El-Sheikh²,3, Ahmed Ragaa Nour Ibrahim ${ }^{4,5}$, Mahmoud El-Daly ${ }^{6}$ \\ 1Department of Pharmaceutical Sciences, College of Clinical Pharmacy, King Faisal University, Al-Ahsa, SAUDI ARABIA. \\ ${ }^{2}$ Department of Pharmacology, Faculty of Medicine, Minia University, El-Minia, EGYPT. \\ ${ }^{3}$ Basic Health Sciences Department, Faculty of Medicine, Princess Nourah bint Abdulrahman University, Riyadh, SAUDI ARABIA. \\ ${ }^{4}$ Department of Clinical Pharmacy, College of Pharmacy, King Khalid University, Abha, SAUDI ARABIA. \\ ${ }^{5}$ Department of Biochemistry, Faculty of Pharmacy, Minia University, El-Minia, EGYPT. \\ ${ }^{6}$ Department of Pharmacology and Toxicology, Faculty of Pharmacy, Minia University, El-Minia, EGYPT.
}

\begin{abstract}
Background: Methotrexate (MTX), a successfully used chemotherapeutic in the treatment of various malignancies and autoimmune diseases, might cause severe nephrotoxicity. Here, we aimed at investigating possible nephroprotective effects of hesperidin (HES), a flavanone present in citrus fruits, against MTX-induced toxicity. Methods: Rats were divided into control, HES, MTX, and MTX/HES groups, where HES was administered in a dose of $100 \mathrm{mg} / \mathrm{kg} /$ day orally for 8 days and MTX in a single i.p. dose of $20 \mathrm{mg} / \mathrm{kg}$ on day 5 of the experiment. Results: Pretreatment with HES significantly improved MTXinduced deteriorated kidney function and structure, as well as reversed MTX effects on renal tumor necrosis factor (TNF)- $\alpha$ level and caspase 3 expression. MTX upregulated renal breast cancer resistance protein (BCRP); an efflux transporter that extrudes MTX from the kidney. Unfortunately, MTX/HES did not show a further increase in BCRP expression but rather showed downregulation. MTX also caused downregulation of renal nuclear factor erythroid 2-related factor 2 (Nrf2) and hemeoxygenase-1 (HO-1) expressions, whereas HES reversed the MTX effect and upregulated renal Nrf2/HO-1. Conclusion: HES conferred protection against MTX-mediated nephrotoxicity, at least in part via anti-inflammatory and anti-apoptotic mechanisms. Nrf2/HO-1 pathway, but not $B C R P$, may have a role in HES-induced nephroprotection against MTX toxicity.
\end{abstract}

Key words: Methotrexate, Hesperidin, Nrf2, HO-1, TNF- $\alpha$, Caspase 3, BCRP.

\section{INTRODUCTION}

For more than six decades, methotrexate (MTX) has been successfully used in a high dose for the treatment of various types of cancers ${ }^{1}$ and in a relatively smaller dose to control autoimmune diseases. ${ }^{2}$ Unfortunately, MTX, especially at high dosages, might cause severe acute renal toxicity in up to $12 \%$ of patients. ${ }^{3}$ The mechanism of MTX-induced nephrotoxicity is not yet fully understood. However, it has been suggested that MTX, especially when given in high dose, may cause glomerular and tubular dysfunction that might lead to the delay of its own elimination and cause its precipitation in high concentrations in renal tubular cells, causing their obstruction and eventually acute renal injury. ${ }^{4}$ Many protein transporters govern the uptake of MTX from the blood through the renal brush border membrane into proximal tubule cells and its subsequent efflux through the apical membrane into the urine. ${ }^{5}$ Breast cancer resistance protein (BCRP) is one of the main renal apical efflux transporters that accept MTX as a substrate. ${ }^{6}$ We have previously shown that MTX-induced renal toxicity might be reversed by resveratrol, at least in part, through modulation of BCRP
Submission Date: 15-09-2021; Revision Date: 24-10-2021; Accepted Date: 17-11-2021

DOI: 10.5530/ijper.55.4.207 Correspondence: Prof. Mohamed A. Morsy Professor, Department of Pharmaceutical Sciences, College of Clinical Pharmacy, King Faisal University, Al-Ahsa 31982 SAUDI ARABIA. Phone no: +966 549672245 E-mail: momorsy@kfu. edu.sa

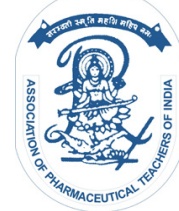

www.ijper.org 
expression in rats in vivo. ${ }^{7}$ In vitro studies also showed that uremic toxins produced during renal damage might further inhibit transport of MTX by BCRP, thus entering in a vicious circuit.

Hesperidin (3,5,7-trihydroxyflavanone 7-rhamnoglucoside; HES), the glycosylation product of hesperitin (40-methoxy30,5,7-trihydroxyflavanone) dietary present almost exclusively in citrus fruits, tomatoes, and some aromatic plants as mint, is reported to have health-promoting effects that may be beneficial in various diseases as hypertension, myocardial infarction, diabetes, neurological disorders, and cancer.' HES is commercially available under different trade names, mostly as a drug combination with the flavonoid diosmin in different ratios, ranging from 1:9 to almost 1:1. Due to its phlebotonic effects, these drug combinations show beneficial effects in improving vascular tone, supporting lymphatic drainage, and strengthening vascular structure; thus, they are indicated for the treatment of acute hemorrhoid, varicose veins, and chronic venous disease. In addition, HES also showed a protective effect against hepatotoxicity induced by another anticancer drug, cisplatin, without affecting its anticancer efficacy. ${ }^{10}$ In the kidney, HES has shown protective effects against renal toxicity caused by 5 -fluorouracil, ${ }^{11}$ cyclophosphamide, ${ }^{12}$ sodium arsenite, ${ }^{13}$ acrylamide, ${ }^{14}$ gentamicin, ${ }^{15}$ and iron. ${ }^{16}$ The aim of the current study is to investigate possible nephroprotective effects of HES on MTX-induced toxicity in rats. In addition, we investigate the involvement of BCRP transporter and/or nuclear factor erythroid 2-related factor 2 (Nrf2)/hemeoxygenase-1 (HO-1) pathways as means of HES-induced protection against MTXinduced nephrotoxicity.

\section{MATERIALS AND METHODS}

\section{Drugs and Chemicals}

HES and MTX (25 mg/ml ampoules) were purchased from Sigma-Aldrich (St. Louis, MO, USA) and Ebewe Co. (Unterach, Austria), respectively. Kits for assessment of blood urea nitrogen (BUN) and creatinine were purchased from Spectrum Diagnostics (Cairo, Egypt). Quantikine rat tumor necrosis factor (TNF)- $\alpha$ enzymelinked immunosorbent assay (ELISA) kit was purchased from R\&D Systems (Minneapolis, MN, USA). All other chemicals were obtained from commercial sources and of analytical grade. RNeasy Purification Reagent and QuantiTect Reverse Transcription Kit were purchased from Qiagen (Valencia, CA, USA). SYBR Green PCR Master Mix was purchased from Applied Biosystems (Foster City, CA, USA).

\section{Animal Study Protocol}

Twenty-four male Wistar rats weighing $190 \pm 10 \mathrm{~g}$ were left to acclimatize for one week before the start of experiments. Rats were housed under standard laboratory animal conditions, at $24 \pm 2{ }^{\circ} \mathrm{C}$, freely accessing standard laboratory animal chow and tap water. Animal protocol ethically followed the Research Ethics Committee guidelines, King Faisal University, which is in accordance with the National Committee of BioEthics (NCBE), KACST, Riyadh, Saudi Arabia. Animals were divided into four groups $(n=6)$. The first group served as control. The second group was treated with freshly prepared HES powder in $0.5 \%$ carboxymethyl cellulose in a dose of $100 \mathrm{mg} / \mathrm{kg} /$ day orally ${ }^{12,13}$ for 8 days. The third group was treated with a single intraperitoneal injection of MTX in a dose of $20 \mathrm{mg} / \mathrm{kg}^{7}$ on day 5 of the experiment. The fourth group received both HES and MTX in combination at the previously indicated dosage regimens.

\section{Sampling Procedures}

After the $8^{\text {th }}$ day of the experiment, animals were sacrificed. Blood samples were collected and centrifuged at $3000 \mathrm{~g}$ for $15 \mathrm{~min}$ to obtain the sera that were aliquoted and stored at $-20^{\circ} \mathrm{C}$ till used. Both kidneys were rapidly extracted, and a longitudinal slice was taken from the right kidney of each animal for histopathological examination. The cortices of the remainder of both kidneys were snap-frozen in liquid nitrogen and kept at $-80^{\circ} \mathrm{C}$. Kidney tissues were homogenized in $20 \% \mathrm{w} / \mathrm{v}$ ice-cold phosphate buffer (0.01 M, pH 7.4). The homogenate was centrifuged at $3000 \mathrm{~g}$ for $15 \mathrm{~min}$ at $4^{\circ} \mathrm{C}$, and the supernatant was obtained, then divided over a number of containers to avoid sample thawing and refreezing and kept at $-80^{\circ} \mathrm{C}$ until used.

\section{Renal Histopathological and Biochemical Assessment}

Longitudinal slice from each rat kidney was fixed in 10\% neutral buffered formalin solution. Samples were then dehydrated in gradual ethanol $(70-100 \%)$ and cleared in xylene. Afterward, tissue samples were embedded in paraffin. Sections of paraffin blocks of $5 \mu \mathrm{m}$ thickness were cut by microtome and mounted on clean glass slides. Staining of the slides was performed using regular hematoxylin and eosin stain for histopathological analysis under a light microscope. Scoring of pathological changes was performed and was qualified as mild, moderate, or severe if less than 25,50 , and $75 \%$ of the total fields tested were distorted, respectively. ${ }^{17}$ As a marker of renal function and nephrotoxicity, biochemical assessment of serum BUN and creatinine using commercially available 


\begin{tabular}{l}
\hline \multicolumn{2}{|c|}{ Table 1: Sequences of rat primers used in real-time polymerase chain reaction determined using } \\
Genbank database.
\end{tabular}

BCRB: Breast cancer resistance protein; Nrf2: Nuclear factor erythroid 2-related factor 2; HO-1: Hemeoxygenase-1.

kits was performed colorimetrically, according to the manufacturer's guidelines.

\section{Assessment of TNF- $\alpha$ in Renal Homogenate Using ELISA Kit}

The level of renal TNF- $\alpha$ was evaluated according to manufacturer's instructions, where $50 \mu \mathrm{l}$ of kidney homogenate was added to $50 \mu \mathrm{l}$ solution of sample diluent and put in a 96-wells ELISA plate, with 2 wells containing sample diluent only without tissue homogenate to serve as blank. After mixing, the plate was incubated for $2 \mathrm{hr}$ at room temperature as a first incubation. Afterward, the ELISA plate was washed 5 times using $400 \mu$ l of diluted washing buffer, followed by drying. An amount of $100 \mu \mathrm{l}$ of rat TNF- $\alpha$ conjugate was dispensed in each well, except for the blank wells, then incubated again and washed as previously described. From each substrate solution, $100 \mu$ l were added to each well; then the plate was covered to protect from light and incubated for $30 \mathrm{~min}$ at room temperature. The reaction was terminated by adding a $100 \mu \mathrm{l}$ stop solution. Using an ELISA plate reader, the plate was read at $450 \mathrm{~nm}$, and the concentration was evaluated via the standard curve.

\section{Real-Time Polymerase Chain Reaction (PCR)}

For assessment of expression of caspase 3, BCRP, Nrf2, and HO-1, real-time PCR was carried out. Using RNeasy purification reagent, total RNA was isolated from kidney tissue homogenate according to the manufacturer's instructions. Denaturation of total renal RNA was performed at $70^{\circ} \mathrm{C}$ for $2 \mathrm{~min}$, which was then reverse-transcribed using QuantiTect Reverse Transcription Kit. The real-time PCR procedures were done using SYBR Green PCR Master Mix together with
$200 \mathrm{ng}$ of each primer. The sequences of the forward and reverse primers of caspase 3, BCRP, Nrf2, and $\mathrm{HO}-1$, as well as $\beta$-actin, are listed in Table 1. PCR reactions comprised of 1 cycle at $95^{\circ} \mathrm{C}$ for $10 \mathrm{~min}$, $94^{\circ} \mathrm{C}$ for $15 \mathrm{~s}$, and 40 cycles at $60^{\circ} \mathrm{C}$ for $1 \mathrm{~min}$, which were performed on a StepOnePlus System (Applied Biosystems). Analysis of data was performed employing ABI Prism 7500 Sequence Detection System software, and quantification was done via version 1.7 Sequence Detection Software from PE Biosystems (Foster City, CA, USA). Normalization of the expression of the studied gene was performed relative to $\beta$-actin utilized as an internal standard, and calculation was performed using the comparative threshold cycle method. ${ }^{18}$

\section{Statistical Analysis}

Data were presented as means \pm SEM, and statistical analysis was performed by GraphPad Prism version 5.00 for Windows (San Diego, CA, USA), via Tukey-Kramer post-analysis test that compares all groups, preceded by one-way analysis of variance (ANOVA). The $P$ value was considered significant if less than 0.05 .

\section{RESULTS}

\section{Effect of HES on Renal Histopathological Changes}

Renal histopathological changes are presented in Figure 1. Both control and the group treated orally with $100 \mathrm{mg} / \mathrm{kg} /$ day of HES for 8 days showed normal glomerular and tubular renal architecture (Figure 1A,B). On the other hand, a single i.p. dose of MTX of $20 \mathrm{mg} / \mathrm{kg}$ caused apparent renal picture distortion (Figure 1C). Scoring of different pathological features showed that the MTX group had mild tubular necrosis, with 

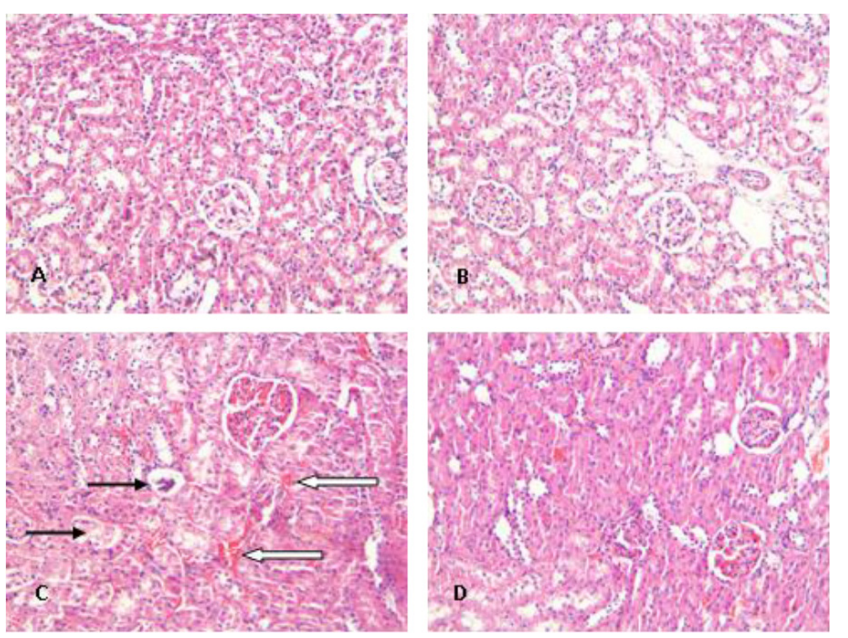

Figure 1: Effect of hesperidin (HES) on histopathological changes of rat kidney in methotrexate (MTX)-induced toxicity. Rat kidney photomicrographs stained by hematoxylin and eosin ( $\times 200)$ of (A) control, (B) HES, (C) MTX and (D) MTX/HES groups. Black arrow, pyknotic distorted glomeruli; white arrow, blood vessel congestion and extravasations. Scoring of histopathological changes is summarized in Table 2.

Table 2: Effect of hesperidin (HES) on histopathological scoring of microscopic renal picture in methotrexate (MTX)-induced nephrotoxicity in rats.

\begin{tabular}{|c|c|c|c|c|}
\hline & $\begin{array}{c}\text { Tubular } \\
\text { necrosis }\end{array}$ & $\begin{array}{c}\text { Glomerular } \\
\text { damage }\end{array}$ & $\begin{array}{c}\text { Congested } \\
\text { blood } \\
\text { vessels }\end{array}$ & $\begin{array}{c}\text { Leucocytic } \\
\text { infiltration }\end{array}$ \\
\hline Control & - & - & - & - \\
\hline HES & - & - & - & - \\
\hline MTX & + & ++ & +++ & ++ \\
\hline MTX/HES & - & - & + & + \\
\hline
\end{tabular}

The $(-)$ was considered normal histological findings. The $(+),(++)$ and $(+++)$ were indicative of mild, moderate and severe histological distortions of less than 25,50 and $75 \%$ in total fields tested, respectively. Results are of sections of renal tissues from each animal $(n=6), 3$ fields/section.

moderate glomerular damage and leucocytic infiltration, accompanied by severely congested blood vessels (Table 2). Pretreatment with HES prior to MTX challenge reversed glomerular and tubular damage to normal picture (Figure 1D), with only mild congested blood vessels and minimal leucocytic infiltrations.

\section{Effect of HES on Renal Function Parameters}

To test kidney function, BUN and creatinine were biochemically assessed in serum. MTX caused a significant increase of both BUN and creatinine levels compared to control (Figure 2A,B). Pretreatment of HES in the MTX-challenged group caused a significant decrease in both BUN and creatinine compared to MTX alone.
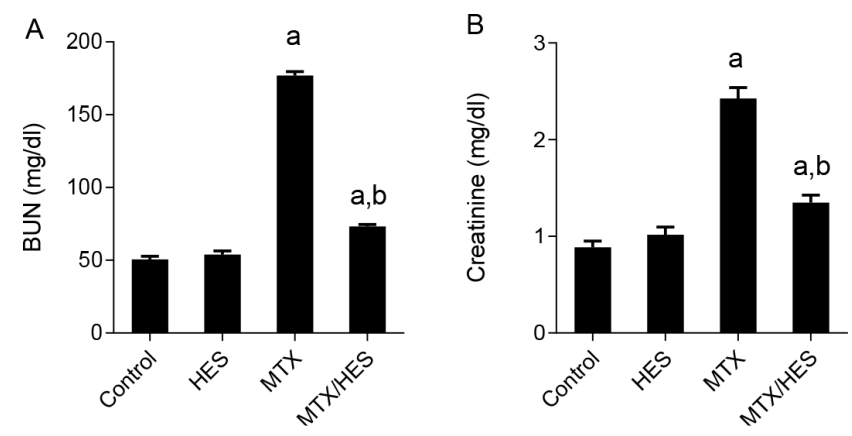

Figure 2: Effect of hesperidin (HES) on blood urea nitrogen (BUN) and creatinine in methotrexate (MTX)-treated rats.

Values represent means \pm SEM $(n=6)$. Significant difference is reported when $P$ is less than 0.05 . "a" Significant difference compared to control and "b" significant difference compared with MTX group.
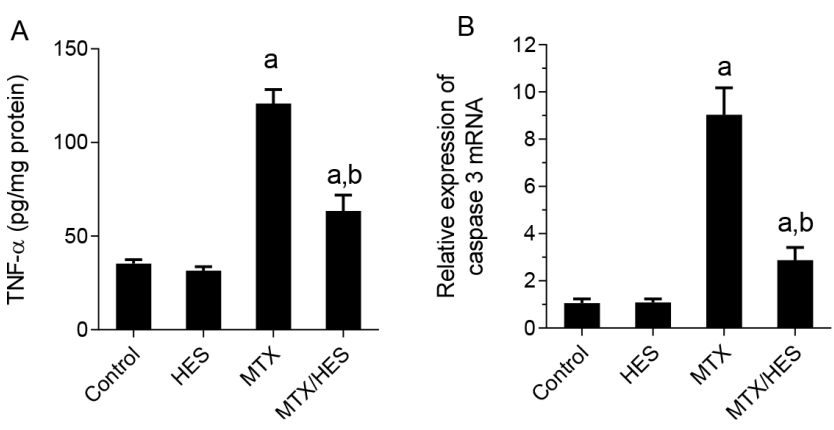

Figure 3: Effect of hesperidin (HES) on renal level of tumor necrosis factor- $\alpha$ (TNF- $\alpha$ ) and caspase 3 mRNA expression in methotrexate (MTX)-treated rats. (A) Renal level of TNF- $\alpha$ assessed by enzyme-linked immunosorbent assay (ELISA) and $(B)$ caspase 3 mRNA relative expression to $\beta$-actin assessed by real-time polymerase chain reaction. Values represent means \pm SEM $(n=6)$. Significant difference is reported when $\boldsymbol{P}$ is less than 0.05 . "a" Significant difference compared with control and " $b$ " significant difference compared with MTX group.

\section{Effect of HES on Renal Inflammatory and Apoptotic Markers}

MTX caused a significant increase in the renal level of TNF- $\alpha$ compared to control (Figure 3A). The group receiving both MTX and HES, however, showed a decrease in renal tissue TNF- $\alpha$ compared to the group receiving MTX alone. Similarly, the pro-apoptotic marker caspase 3, determined via real-time PCR, in the MTX-treated group showed a significant increase in renal expression compared to control (Figure 3B). On the other hand, MTX/HES concomitantly treated group showed a decrease in renal expression of caspase 3 compared to the sole MTX-treated group.

\section{Effect of HES on Renal BCRP, Nrf2, and HO-1 mRNA Expression}

Expression of BCRP in the kidney was not affected by sole treatment with HES at an oral dose of $100 \mathrm{mg} / \mathrm{kg}$ 
for 8 days (Figure 4). However, MTX-treated rats showed significantly increased expression of renal BCRP compared to control. Pretreatment of MTX-challenged rats with HES, however, caused a significant reduction of BCRP expression compared to the MTX-treated group. Expression of both Nrf2 and HO-1 was not affected by sole treatment of HES compared to control (Figure 5). MTX treatment, on the other hand, caused a significant decrease in renal expression of both Nrf2 and HO-1 compared to control. Treatment with HES in MTX-challenged rats, however, caused a significant increase of both Nrf2 and HO-1 compared to MTX alone, to levels insignificant from that of control.

\section{DISCUSSION}

In the current study, MTX caused nephrotoxicity, as evident by significantly increasing kidney function tests and disrupting normal renal histological architecture, which is in line with our previous studies. ${ }^{7,9,20}$ To investigate the mechanisms by which MTX may induce nephrotoxicity, the current study showed that MTX caused an increase in renal levels of TNF- $\alpha$ and caspase 3 , indicating the stimulation of inflammatory and apoptotic pathways as possible mechanisms of MTXinduced nephrotoxicity, which is also in agreement with previous results. ${ }^{19-21}$

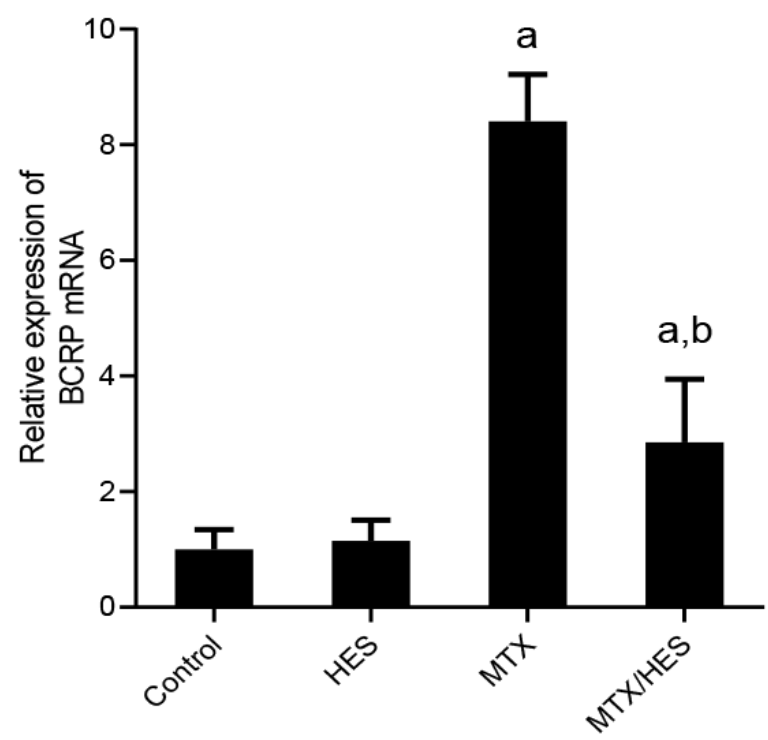

Figure 4: Effect of hesperidin (HES) on renal level of breast cancer resistance protein (BCRP) mRNA expression in methotrexate (MTX)-treated rats. BCRP mRNA relative expression to $\beta$-actin was assessed by real-time polymerase chain reaction. Values represent means \pm SEM $(n=6)$. Significant difference is reported when $P$ is less than 0.05 . "a" Significant difference compared with control and " $b$ " significant difference compared with MTX group.
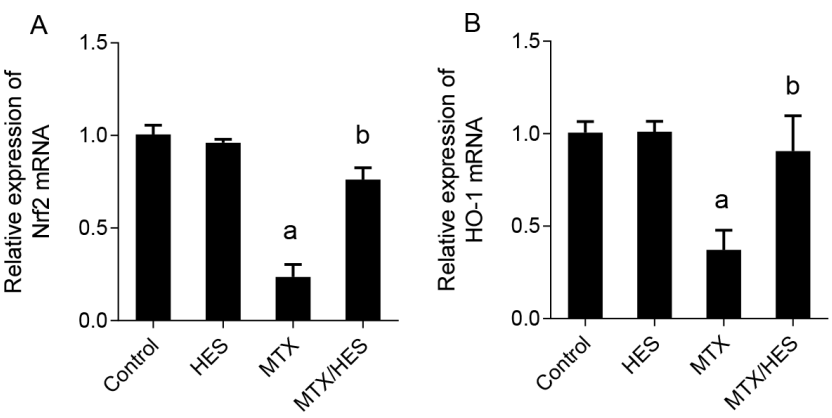

Figure 5: Effect of hesperidin (HES) on renal mRNA expression of (A) nuclear factor erythroid 2-related factor 2 (Nrf2) and

(B) hemoxygenase-1 (HO-1) in methotrexate (MTX)-treated rats. The mRNA relative expression to $\beta$-actin assessed by real-time polymerase chain reaction. Values represent means \pm SEM $(n=6)$. Significant difference is reported when $P$ is less than 0.05. "a" Significant difference compared with control and "b" significant difference compared with MTX group.

Pretreatment with HES improved renal function in MTX-treated rats, as evident by the significant decrease in BUN and creatinine. Consistent with our results, several previous studies showed that HES improved renal function impairment caused by 5 -fluorouracil, ${ }^{11}$ cyclophosphamide, ${ }^{12}$ sodium arsenite, ${ }^{13}$ acrylamide, ${ }^{14}$ gentamicin, ${ }^{15}$ and iron. $^{16}$ In addition, pretreatment with HES tremendously improved renal pathological distortions induced by MTX. Similar histopathological findings of HES-induced nephroprotection were reported in renal damage induced by 5 -fluorouracil, ${ }^{11}$ cyclophosphamide, ${ }^{12}$ sodium arsenite, ${ }^{13}$ gentamicin, ${ }^{15}$ and cisplatin. ${ }^{22}$

In the current study, HES showed anti-inflammatory properties, as it ameliorated the increased TNF- $\alpha$ level induced by MTX in the kidney. Previous studies showed that HES may possess a similar anti-inflammatory effect and may decrease renal tissue levels of TNF- $\alpha$ induced by cyclophosphamide, ${ }^{12}$ sodium arsenite, ${ }^{13}$ gentamicin,${ }^{15}$ and sodium fluoride. ${ }^{23}$ Besides the kidney, HES was also reported to have a comparable anti-inflammatory effect in other organs like the liver, as it decreased TNF- $\alpha$ in sodium arsenite ${ }^{13}$ and sodium fluoride ${ }^{23}$ induced hepatotoxicity. In addition, HES decreased TNF- $\alpha$ in sodium arsenite-induced cardiotoxicity and neurotoxicity. ${ }^{24} \mathrm{TNF}-\alpha$ binds to its receptors and stimulates receptor-dependent pathway of apoptosis through activation of caspases, including caspase $3 .{ }^{25}$ Thus, in addition to its anti-inflammatory effects, HES, in the current study, showed an anti-apoptotic effect, as it decreased the renal expression of the pro-apoptotic marker; caspase 3, induced by MTX. Comparable results on caspase 3 were previously documented in previous studies, where HES had an anti-apoptotic 
effect in nephrotoxicity induced by 5 -fluorouracil, ${ }^{11}$ cyclophosphamide, ${ }^{12}$ sodium arsenite, ${ }^{13}$ cisplatin. ${ }^{22}$ and trichloroethylene. ${ }^{26}$ Other than the kidney, HES was also reported to decrease hepatic caspase 3 induced by sodium arsenite ${ }^{13}$ and carbon tetrachloride. ${ }^{27}$ In addition, HES diminished caspase 3 in sodium arsenite-induced cardiotoxicity and neurotoxicity. ${ }^{24}$ These multi-organ results support that HES might have generalized multiorgan anti-inflammatory and anti-apoptotic properties.

In the current study, MTX caused more than an 8-fold increase in renal expression of BCRP, which is in line with our previous results. ${ }^{7}$ Interestingly, HES in the present study, instead of increasing renal BCRP, rather, it decreased it, suggesting no role of BCRP in HESinduced nephroprotection against MTX. Still, in the kidney, MTX is accepted as a substrate by a number of apical transporters, other than BCRP, that actively efflux MTX at the expense of ATP, including a number of multidrug resistance proteins. ${ }^{5,28}$ It is possible that HES might have mediated its nephroprotective effects by affecting the expression of other transporters capable of modulating MTX renal excretion, which is mere speculation in need of further investigations.

$\mathrm{Nrf2/HO}-1$ pathway is one of the main mechanisms that combat oxidative stress. As an upper stream regulator, Nrf2 binds HO-1 antioxidant responsive element, initiating a plethora of genetic transcriptional activation that results in the production of antioxidant and cellular protective proteins that eventually ameliorate oxidative stress. ${ }^{29}$ In the present study, MTX downregulated the expression of both Nrf2 and HO-1 in rat kidneys. Previous studies showed similar MTX downregulation of $\mathrm{Nrf2/HO}-1$ pathway in kidney ${ }^{30,31}$ In the current study, HES reversed the down regulatory effect of MTX and caused upregulation of both Nrf2 and HO-1 renal expression. Interestingly, in the current study, HES alone without MTX challenge had no effect on either renal Nrf2 or HO-1 expression. The reported effects of HES on the Nrf2/HO-1 pathway are controversial. In the kidney, HES, with or without challenge, was reported to upregulate renal $\mathrm{Nrf} 2$ and/or HO-1 in a model of diethylnitrosamine/carbon tetrachlorideinduced kidney repercussions. ${ }^{32}$ On the other hand, only HES+gentamicin, not HES alone, could induce the expression of $\mathrm{Nrf2}$ and/or HO-1 in gentamicininduced nephrotoxicity model. ${ }^{15}$

It is possible that HES-induced upregulation of antioxidant Nrf2/HO-1 pathway in MTX-challenged rat kidney in the current study had triggered the amelioration of inflammation and/or apoptosis, depicted by the decrease in renal levels of TNF- $\alpha$ and caspase 3 expression. We have previously shown that the modulation of HO-1 expression and/or activity may affect renal levels of TNF- $\alpha$ and caspase $3,,^{33}$ suggesting that their upregulation by MTX possess an upper stream factor that may govern renal inflammation and apoptosis, thus inducing nephrotoxicity. The cross-talk between these pathways has indeed been previously reported, where upregulation of renal Nrf2/ HO-1 was concomitantly encountered together with a decrease in renal TNF- $\alpha$ by ergothioneine in cisplatininduced nephrotoxicity, ${ }^{34}$ by phenethyl isothiocyanate in streptozotocin-induced diabetic nephropathy, ${ }^{35}$ and by quercetin on copper sulfate-induced nephrotoxicity. ${ }^{36}$ Similar findings have been reported linking the upregulation of renal $\mathrm{Nrf2/HO}-1$ to caspase 3 downregulation by ergothioneine in cisplatin-induced nephrotoxicity, ${ }^{34}$ by camel milk in cyclosporine-induced nephrotoxicity, ${ }^{37}$ and by thymoquinone in diclofenacinduced acute kidney injury. ${ }^{38}$ However, despite all previous reports, it is extremely difficult to hypothesize a cause-effect relationship between these complex pathways. ${ }^{39}$

\section{CONCLUSION}

Despite not having any favorable effects on renal BCRP expression, still HES pretreatment succeeded in ameliorating MTX-induced nephrotoxicity. The mechanisms involved included, at least in part, reversing renal inflammatory and apoptotic status deteriorated by MTX. In addition, HES reversed renal downregulation of Nrf2/HO-1 expression by MTX.

\section{ACKNOWLEDGEMENT}

This research was funded by the Deanship of Scientific Research at King Faisal University, Al-Ahsa, Saudi Arabia (Project number 150201).

\section{CONFLICT OF INTEREST}

The authors declare no conflict of interest.

\section{ABBREVIATIONS}

BCRP: Breast cancer resistance protein; BUN: Blood urea nitrogen; ELISA: Enzyme-linked immunosorbent assay; HES: Hesperidin; HO-1: Hemeoxygenase-1; MTX: Methotrexate; Nrf2: Nuclear factor erythroid 2-related factor 2; PCR: Polymerase chain reaction; TNF- $\alpha$ : Tumor necrosis factor- $\alpha$. 


\section{REFERENCES}

1. Taylor ZL, Vang J, Lopez-Lopez E, Oosterom N, Mikkelsen T, Ramsey LB. Systematic review of pharmacogenetic factors that influence high-dose methotrexate pharmacokinetics in pediatric malignancies. Cancers (Basel). 2021;13(11):2837. doi: 10.3390/cancers13112837, PMID 34200242.

2. Lucas CJ, Dimmitt SB, Martin JH. Optimising low-dose methotrexate for rheumatoid arthritis-A review. Br J Clin Pharmacol. 2019;85(10):2228-34. doi: 10.1111/bcp.14057, PMID 31276602.

3. Howard SC, McCormick J, Pui CH, Buddington RK, Harvey RD. Preventing and managing toxicities of high-dose methotrexate. Oncologist. 2016;21(12):1471-82. doi: 10.1634/theoncologist.2015-0164, PMID 27496039.

4. Sharbaf FG, Farhangi H, Assadi F. Prevention of chemotherapy-induced nephrotoxicity in children with cancer. Int J Prev Med. 2017;8:76. doi: 10.4103/ijpvm.IJPVM_40_17, PMID 29114374.

5. El-Sheikh AA, Masereeuw R, Russel FG. Mechanisms of renal anionic drug transport. Eur J Pharmacol. 2008;585(2-3):245-55. doi: 10.1016/j. ejphar.2008.02.085, PMID 18417112.

6. Volk EL, Schneider E. Wild-type breast cancer resistance protein (BCRP/ $A B C G 2)$ is a methotrexate polyglutamate transporter. Cancer Res. 2003;63(17):5538-43. PMID 14500392.

7. El-Sheikh AA, Morsy MA, Al-Taher AY. Protective mechanisms of resveratrol against methotrexate-induced renal damage may involve BCRP/ABCG2. Fundam Clin Pharmacol. 2016;30(5):406-18. doi: 10.1111/fcp.12205, PMID 27213989.

8. Mutsaers HA, Van den Heuvel LP, Ringens LH, Dankers AC, Russel FG, Wetzels JF, et al. Uremic toxins inhibit transport by breast cancer resistance protein and multidrug resistance protein 4 at clinically relevant concentrations. PLOS ONE. 2011;6(4):e18438. doi: 10.1371/journal.pone.0018438, PMID 21483698.

9. Li C, Schluesener H. Health-promoting effects of the citrus flavanone hesperidin. Crit Rev Food Sci Nutr. 2017;57(3):613-31. doi: 10.1080/10408398.2014.906382, PMID 25675136.

10. Omar HA, Mohamed WR, Arafa el-SA, Shehata BA, El Sherbiny GA, Arab $\mathrm{HH}$, et al. Hesperidin alleviates cisplatin-induced hepatotoxicity in rats without inhibiting its antitumor activity. Pharmacol Rep. 2016;68(2):349-56. doi: 10.1016/j.pharep.2015.09.007, PMID 26922538.

11. Gelen V, Şengül E, Yıldırım S, Senturk E, Tekin S, Kükürt A. The protective effects of hesperidin and curcumin on 5-fluorouracil-induced nephrotoxicity in mice. Environ Sci Pollut Res Int. 2021;28(34):47046-55. doi: 10.1007/s11356021-13969-5, PMID 33886055.

12. Fouad AA, Abdel-Gaber SA, Abdelghany MI. Hesperidin opposes the negative impact of cyclophosphamide on mice kidneys. Drug Chem Toxicol. 2021;44(3):223-8. doi: 10.1080/01480545.2018.1560467, PMID 30889984

13. Turk E, Kandemir FM, Yildirim S, Caglayan C, Kucukler S, Kuzu M. Protective effect of hesperidin on sodium arsenite-induced nephrotoxicity and hepatotoxicity in rats. Biol Trace Elem Res. 2019;189(1):95-108. doi: 10.1007/s12011-018-1443-6, PMID 30066062.

14. Hamdy SM, Shabaan AM, Abdel Latif AKM, Abdel-Aziz AM, Amin AM Protective effect of hesperidin and tiger nut against acrylamide toxicity in female rats. Exp Toxicol Pathol. 2017;69(8):580-8. doi: 10.1016/j. etp.2017.05.004, PMID 28551406.

15. Subramanian P, Anandan R, Jayapalan JJ, Hashim OH. Hesperidin protects gentamicin-induced nephrotoxicity via $\mathrm{Nr} 2 / \mathrm{HO}-1$ signaling and inhibits inflammation mediated by NF-KB in rats. J Funct Foods. 2015;13:89-99. doi: 10.1016/j.jf.2014.12.035.

16. Pari L, Karthikeyan A, Karthika P, Rathinam A. Protective effects of hesperidin on oxidative stress, dyslipidaemia and histological changes in iron-induced hepatic and renal toxicity in rats. Toxicol Rep. 2015;2:46-55. doi: 10.1016/j. toxrep.2014.11.003, PMID 28962336.

17. El-Sheikh AA, Morsy MA, Mahmoud MM, Rifaai RA, Abdelrahman AM. Effect of coenzyme-q10 on doxorubicin-induced nephrotoxicity in rats. Adv Pharmacol Sci. 2012;2012:981461. doi: 10.1155/2012/981461.

18. Livak KJ, Schmittgen TD. Analysis of relative gene expression data using real-time quantitative PCR and the 2(-Delta Delta $\mathrm{C}(\mathrm{T})$ ) method. Methods. 2001;25(4):402-8. doi: 10.1006/meth.2001.1262, PMID 11846609.
19. El-Sheikh AA, Morsy MA, Abdalla AM, Hamouda AH, Alhaider IA. Mechanisms of thymoquinone hepatorenal protection in methotrexate-induced toxicity in rats. Mediators Inflamm. 2015;2015:859383. doi: 10.1155/2015/859383.

20. Ibrahim MA, El-Sheikh AA, Khalaf HM, Abdelrahman AM. Protective effect of peroxisome proliferator activator receptor (PPAR)-alpha and -gamma ligands against methotrexate-induced nephrotoxicity. Immunopharmacol Immunotoxicol. 2014;36(2):130-7. doi: 10.3109/08923973.2014.884135, PMID 24521009.

21. Osman AT, Sharkawi SMZ, Hassan MIA, Abo-Youssef AM, Hemeida RAM. Empagliflozin and neohesperidin protect against methotrexate-induced renal toxicity via suppression of oxidative stress and inflammation in male rats. Food Chem Toxicol. 2021;155:112406. doi: 10.1016/j.fct.2021.112406. PMID 34256053.

22. Sahu BD, Kuncha M, Sindhura GJ, Sistla R. Hesperidin attenuates cisplatininduced acute renal injury by decreasing oxidative stress, inflammation and DNA damage. Phytomedicine. 2013;20(5):453-60. doi: 10.1016/j. phymed.2012.12.001, PMID 23353054.

23. Caglayan C, Kandemir FM, Darendelioğlu E, Küçükler S, Ayna A. Hesperidin protects liver and kidney against sodium fluoride-induced toxicity through antiapoptotic and anti-autophagic mechanisms. Life Sci. 2021;281:119730. doi: 10.1016/j.lfs.2021.119730.

24. Kuzu M, Kandemir FM, Yıldırım S, Çağlayan C, Küçükler S. Attenuation of sodium arsenite-induced cardiotoxicity and neurotoxicity with the antioxidant, anti-inflammatory, and antiapoptotic effects of hesperidin. Environ Sci Pollut Res Int. 2021;28(9):10818-31. doi: 10.1007/s11356-020-11327-5, PMID 33099738.

25. Kaushal GP, Kaushal V, Hong X, Shah SV. Role and regulation of activation of caspases in cisplatin-induced injury to renal tubular epithelial cells. Kidney Int. 2001;60(5):1726-36. doi: 10.1046/j.1523-1755.2001.00026.x, PMID 11703590.

26. Siddiqi A, Nafees S, Rashid S, Sultana S, Saidullah B. Hesperidin ameliorates trichloroethylene-induced nephrotoxicity by abrogation of oxidative stress and apoptosis in Wistar rats. Mol Cell Biochem. 2015;406(1-2):9-20. doi: 10.1007/ s11010-015-2400-8, PMID 25994504.

27. Çetin A, Çiftçi O, Otlu A. Protective effect of hesperidin on oxidative and histological liver damage following carbon tetrachloride administration in Wistar rats. Arch Med Sci. 2016;12(3):486-93. doi: 10.5114/aoms.2015.49484, PMID 27279838.

28. Ivanyuk A, Livio F, Biollaz J, Buclin T. Renal drug transporters and drug interactions. Clin Pharmacokinet. 2017;56(8):825-92. doi: 10.1007/s40262017-0506-8, PMID 28210973

29. Ndisang JF. Synergistic interaction between heme oxygenase (HO) and nuclear-factor E2- related factor-2 (Nrf2) against oxidative stress in cardiovascular related diseases. Curr Pharm Des. 2017;23(10):1465-70. doi: 10.2174/1381612823666170113153818, PMID 28088909.

30. Aladaileh SH, Hussein OE, Abukhalil MH, Saghir SAM, Bin-Jumah M, Alfwuaires MA, et al. Formononetin upregulates Nrf2/HO-1 signaling and prevents oxidative stress, inflammation, and kidney injury in methotrexateinduced rats. Antioxidants (Basel). 2019;8(10):430. doi: 10.3390/ antiox8100430, PMID 31561418.

31. Shalaby YM, Menze ET, Azab SS, Awad AS. Involvement of Nrf2/HO-1 antioxidant signaling and NF-KB inflammatory response in the potential protective effects of vincamine against methotrexate-induced nephrotoxicity in rats: Cross talk between nephrotoxicity and neurotoxicity. Arch Toxicol. 2019;93(5):1417-31. doi: 10.1007/s00204-019-02429-2, PMID 31020375.

32. Aly MS, Galaly SR, Moustafa N, Mohammed HM, Khadrawy SM, Mahmoud AM. Hesperidin protects against diethylnitrosamine/carbon tetrachlorideinduced renal repercussions via up-regulation of $\mathrm{Nrf2} / \mathrm{HO}-1$ signaling and attenuation of oxidative stress. J Appl Pharm Sci. 2017;7(11):7-14.

33. Ali MAM, Heeba GH, El-Sheikh AAK. Modulation of heme oxygenase-1 expression and activity affects streptozotocin-induced diabetic nephropathy in rats. Fundam Clin Pharmacol. 2017;31(5):546-57. doi: 10.1111/fcp.12296, PMID 28543864.

34. Salama SA, Abd-Allah GM, Mohamadin AM, Elshafey MM, Gad HS Ergothioneine mitigates cisplatin-evoked nephrotoxicity via targeting Nrf2, NF-KB, and apoptotic signaling and inhibiting Y-glutamyl transpeptidase. Life Sci. 2021;278:119572. doi: 10.1016/j.lfs.2021.119572. 
35. Eisa NH, Khodir AE, El-Sherbiny M, Elsherbiny NM, Said E. Phenethyl isothiocyanate attenuates diabetic nephropathy via modulation of glycative/ oxidative/inflammatory signaling in diabetic rats. Biomed Pharmacother. 2021;142:111666. doi: 10.1016/j.biopha.2021.111666.

36. Peng X, Dai C, Zhang M, Das Gupta S. Molecular mechanisms underlying protective role of quercetin on copper sulfate-induced nephrotoxicity in mice. Front Vet Sci. 2020;7:586033. doi: 10.3389/fvets.2020.586033.

37. Arab HH, Eid AH, Gad AM, Yahia R, Mahmoud AM, Kabel AM. Inhibition of oxidative stress and apoptosis by camel milk mitigates cyclosporine-induced nephrotoxicity: Targeting Nrf2/HO-1 and AKT/eNOS/NO pathways. Food Sci Nutr. 2021;9(6):3177-90. doi: 10.1002/fsn3.2277, PMID 34136182.

38. Hashem KS, Abdelazem AZ, Mohammed MA, Nagi AM, Aboulhoda BE, Mohammed ET, et al. Thymoquinone alleviates mitochondrial viability and apoptosis in diclofenac-induced acute kidney injury (AKI) via regulating Mfn2 and miR-34a mRNA expressions. Environ Sci Pollut Res Int. 2021;28(8):10100-13. doi: 10.1007/s11356-020-11313-x, PMID 33165700.

39. Taye A, El-Sheikh AA. Lectin-like oxidized low-density lipoprotein receptor 1 pathways. Eur J Clin Investig. 2013;43(7):740-5. doi: 10.1111/eci.12092, PMID 23594336.

\section{PICTORIAL ABSTRACT}

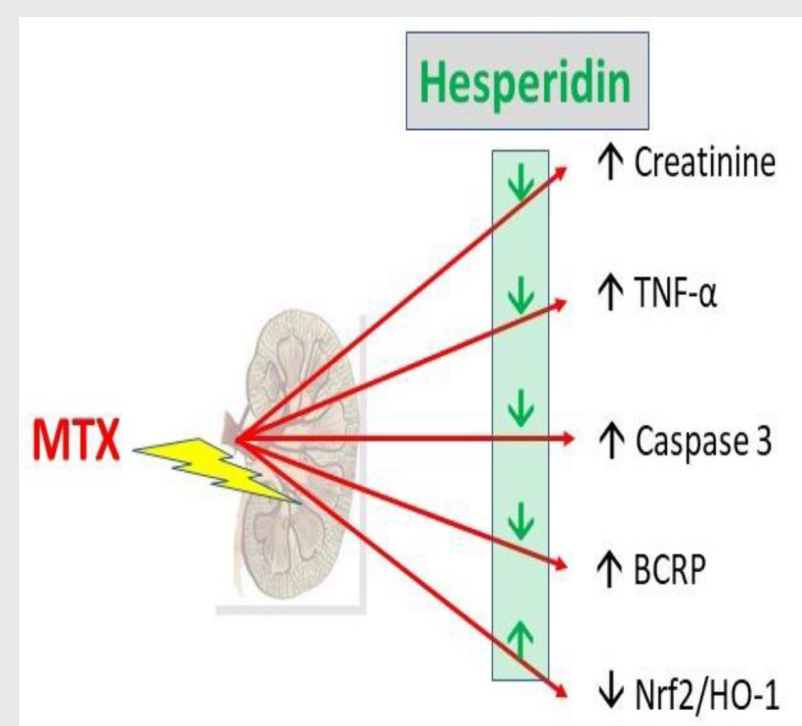

\section{SUMMARY}

Hesperidin protects against methotrexate-induced nephrotoxicity. Mechanisms involved is downregulation of $\mathrm{TNF}-\alpha$ and caspase 3. Methotrexate induced renal BCRP expression. Concomitant hesperidin didn't further induce, but inhibited renal BCRP expression. Hesperidin induced Nrf2/HO-1 pathway in methotrexate-induced nephrotoxicity.

Cite this article: Morsy MA, El-Sheikh AAK, Ibrahim ARN, El-Daly M. Protection of Hesperidin against MethotrexateInduced Nephrotoxicity may be Mediated by Nrf2/HO-1 Pathway. Indian J of Pharmaceutical Education and Research. 2021;55(4):1066-73. 